\title{
Advanced Motorcycle-Infrastructure-Driver Roll Angle Profile for Loss Control Prevention
}

\author{
H. Slimi, H. Arioui, L. Nouveliere and S. Mammar
}

\begin{abstract}
In this paper, we present a new method for the calculation of the maximum roll angle of a motorcycle authorized during driving in curve situation. The proposed approach takes into account the three elements of the driving situation which are the vehicle the driver and the infrastructure. The vehicle dynamics are represented by a dynamic four degrees of freedom model which include longitudinal and lateral dynamics of the motorcycle. The driver behaviour considers his ability in deceleration taking into account his mobilized friction while the infrastructure characteristics introduce a precise definition of the road geometry and the maximal available friction.
\end{abstract}

\section{INTRODUCTION}

A precise description of the infrastructure and more accurate vehicle dynamic characteristics make possible to consider the development of a more relevant warning system.

This paper presents a computational method of the highest roll angle authorized by a motorcycle for driving the upcoming curve. The interaction of the three actors which are the vehicle, the driver and the infrastructure are taken into account. The vehicle dynamics are represented by a dynamic model which includes longitudinal load transfer. The driver behaviour considers his ability in deceleration while the infrastructure characteristics introduce a precise definition of the road geometry (curvature, slope and road bank angle) and the maximal available friction.

We also study the influence of the various parameters of the infrastructure and the vehicle dynamics on the calculated roll angle profile. We present and discuss then the simulation results.

\section{PROBLEM STATEMENT: ARCHITECTURE FOR THE WARNING SYSTEM}

The long-term objective of this study is to present a proposal for assistance to drivers of two-wheeled vehicles by preventing them sufficiently in advance to anticipate dangerous situations (figure 1) [11]. Recall that since 20 years there are 1000 to 1600 killed each year caused by motorcycle, either a probability of death 21 times higher than the other road users [12].

The main idea is to study and develop a system for motorcycle safety which detects and alerts the driver for the loss-control situations [11]. In figure 1 we present a proposal architecture for assistance to drivers of two-wheeled vehicle.

The warning system is interacting with the triplet D-V-I via a HMI interface. In the triplet D-V-I, the driver influences the dynamic's motorcycle by its inputs (Torque, roll and lean

H. Slimi, H. Arioui, L. Nouveliere and S. Mammar are with IBISC-CNRS Fre 3190 Lab. of Evry Val d'Essonne University, 91020 Evry Cedex, France hamid.slimidibisc.fr

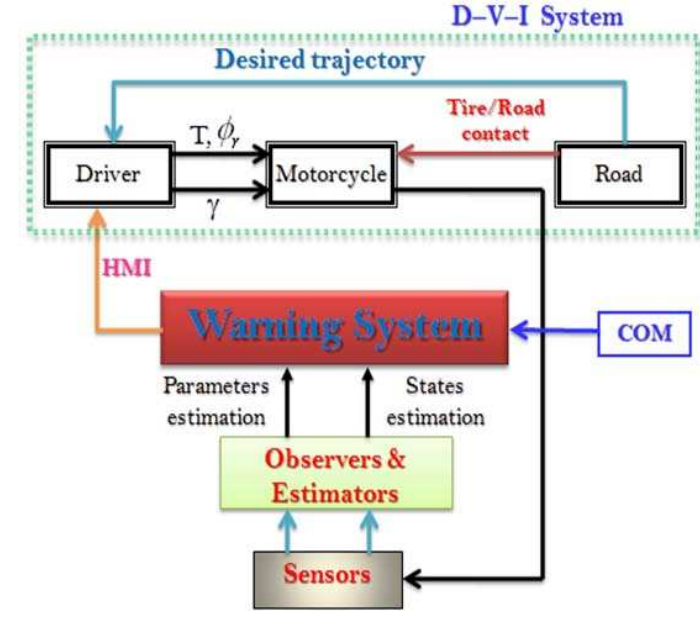

Fig. 1. Architecture of the warning system [11]

angle), the road also affects the vehicle through tire/road contact, and dictates the desired trajectory for a driver.

The warning system exploits the information given by different physical sensors, for known the changing dynamic state and the variation of dynamic parameters. However, these states and dynamic parameters are not directly measurable: sensors do not exist yet, or they are prohibitively expensive; in this case we can use the observers.

This paper is dedicated only to the generation of a maximum roll angle profile authorized, in cornering situation, which will be a benchmarked limit for the warning system. An alarm is delivered when this limit is reached.

\section{EXISTING DESIRED ROLL ANGLE MODELS}

The desired safe roll angle in a curve depends on the road geometry, the surface conditions, the driver skills and the rollover stability of the vehicle [1], [3]. The geometric factors of a curve that are always fixed are its radius of curvature, its slop and its superelevation or banking. The other roaddependent factor is the maximum lateral friction factor that can be generated by the road surface. The friction factor can vary from vehicle to vehicle with (1) the temperature of the surface, (2) condition of the road's surface and (3) the tires and longitudinal speed of the vehicle [1].

If we neglect any others parameter than the curvature $\rho$, the safe roll angle at which a vehicle can be kept on the road while moving at a constant longitudinal speed on a circular section is given by [1], [4]: 


$$
\phi_{\text {des }}=\arctan \left(\frac{v_{x}^{2} \rho}{g}\right)=\arctan \left(\frac{v_{x} \dot{\psi}_{\text {des }}}{g}\right)
$$

Where $g$ is the gravity, $v_{x}$ is the longitudinal speed and $\dot{\psi}_{\text {des }}$ is the desired yaw rate. Due to the lack of any other road geometry attribute than curvature in currently available map databases [10].

One can go one step further by taking into account the superelevation $\phi_{r}$. Evangelou and al in [1], propose a formula (2) for the maximum safe roll angle in curve, which takes into account the geometry of the road in particular the banking.

$$
\left|\phi_{\text {des }}-\phi_{r}\right|<\arctan \left(\mu_{\text {limit }}\right)+\arcsin \left(\frac{\mu_{\text {limit }} R}{l_{0} \sqrt{1+\mu_{\text {limit }}^{2}}}\right)
$$

This simplifies to

$$
\left|\phi_{\text {des }}-\phi_{r}\right|<\arctan \left(\mu_{\text {limit }}\right)
$$

$\mu_{\text {limit }}$ is the friction limit, $R$ is the tire crown radius, and the distance between a motorcycle's mass center and the center of the tire crown is $l_{0}$. The distance $l_{0}+R$ is the height of the machine's mass center [1] above a level road surface when it is in its nominal configuration (vertical position).

The determination of the maximum authorized roll angle in a curve is generally based on the information on lateral acceleration [5]. These constraints express in particular, the maximum road curvature according to acceptable maximum lateral acceleration is between $0.2 \mathrm{~g} \mathrm{~m} / \mathrm{s}^{2}$ and $0.4 \mathrm{~g} \mathrm{~m} / \mathrm{s}^{2}$.

\section{PROPOSED APPROACH FOR ROLL ANGLE PROFILE GENERATION}

The model of the maximum safe roll angle authorized in curve developed by Evangelou and al in [1] (formula (2)) is more precise compared with the one given by formula (1) because the banking and the friction limit are taken into account. However, the vehicle dynamics and some attributes of the road, as the slope and the driver behaviour are not entirely taken into account. The following enhancements are considered below.

The idea is to use the formula (1) $\phi_{\text {des }}=\arctan \left(\frac{v_{x}^{2} \rho}{g}\right)$ that is function of speed, then, we determine in the next section the maximum speed authorized for negotiate a turn without loss control, taking into account the dynamics of the vehicle, the driver mobilized friction and the geometry of the road.

\section{A. Motorcycle Dynamic Model}

We chose a dynamic model of the vehicle which takes into account at the same time the forces at the tire/road contact point and the load transfer due to the longitudinal accelerations [2], [6], [7], [8]. Other original models exist [13]. We will describe the vehicle's movements.

The longitudinal dynamics are given by two equations which represent the longitudinal translation and the roll rate moment:

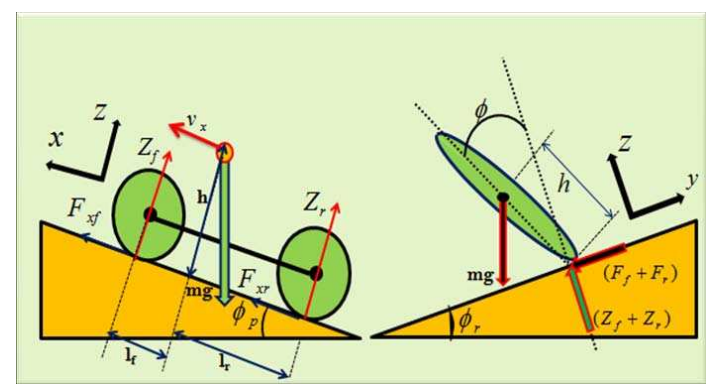

Fig. 2. Parameters and Forces of the Motorcycle

$$
\left\{\begin{array}{l}
M\left(\dot{v}_{x}-h \phi \ddot{\psi}\right)=\underbrace{\left(F_{x f}+F_{x r}\right)}_{F_{\text {long }}}+M \cdot g \cdot \sin \left(\phi_{p}\right) \\
I_{x} \ddot{\phi}=M g h \phi-\left(F_{f}+F_{r}\right) h
\end{array}\right.
$$

Where $F_{\text {long }}=\left(F_{x f}+F_{x r}\right)$ represents the longitudinal force acting on the tires (front and rear) [10]. In the same way, the lateral motion is described by two equations which represent the effect of the lateral forces on the lateral movement and the yaw rate $\dot{\psi}$ :

$$
\left\{\begin{array}{l}
M\left(\ddot{y}+\dot{\psi} v_{x}+h \ddot{\phi}\right)=\underbrace{\left(F_{f}+F_{r}\right)}_{F_{\text {lat }}}-M \cdot g \cdot \cos \left(\phi_{p}\right) \sin \left(\phi_{r}\right) \\
I_{z} \ddot{\psi}=l_{f} \cdot F_{f}+l_{r} \cdot F_{r}
\end{array}\right.
$$

Where $y$ is the lateral displacement, $I_{z}$ is the inertial moment, around the $z$-axis, $\phi$ is the roll angle.

$h, l_{f}, l_{r}$ parameters defining in figure 2. $F_{l a t}=\left(F_{f}+F_{r}\right)$ is the lateral force exerted on the front and rear tires.

1) Steady State Equilibrium: The tilt (roll) stability of the motorcycle is ensured if the sum of the steady state moments acting at the center of gravity of the vehicle is zero. That is to say: $I_{x} \ddot{\phi}=0 \Rightarrow \ddot{\phi}=0$.

One can express the lateral forces on the front and rear wheels using the system equation (4) as follows:

$$
\left\{\begin{array}{l}
F_{f}=\frac{1}{l_{f}+l_{r}}\left(l_{r} \cdot M \cdot \ddot{y}+I_{z} \ddot{\psi}+l_{r} \cdot M \cdot g \cdot \sin \left(\phi_{r}\right)\right) \\
F_{r}=\frac{1}{l_{f}+l_{r}}\left(l_{f} \cdot M \cdot \ddot{y}-I_{z} \ddot{\psi}+l_{f} \cdot M \cdot g \cdot \sin \left(\phi_{r}\right)\right)
\end{array}\right.
$$

In dynamic conditions, load can transfer to the front wheels during braking and on the rear wheels during acceleration. When driver accelerates normal forces on the two tires can be obtained as follows:

$$
\left\{\begin{array}{l}
Z_{f}=\frac{1}{l_{f}+l_{r}}\left(l_{r} \cdot M \cdot g \cdot \cos \left(\phi_{r}\right) \cdot \cos \left(\phi_{p}\right)-h \cdot F_{\text {long }}\right) \\
Z_{r}=\frac{1}{l_{f}+l_{r}}\left(l_{f} \cdot M \cdot g \cdot \cos \left(\phi_{r}\right) \cdot \cos \left(\phi_{p}\right)+h \cdot F_{\text {long }}\right)
\end{array}\right.
$$




\section{B. Tire Road Friction}

The tire is one of the main components of the vehicle. Indeed, it represents the interface of the aforementioned with the external environment which is the road. It transmits the guidance and braking/traction efforts [5]. The tire dynamic behaviour is very complex and is linear only under certain restricted conditions of operation. One observes various phenomena like skidding and blocking.

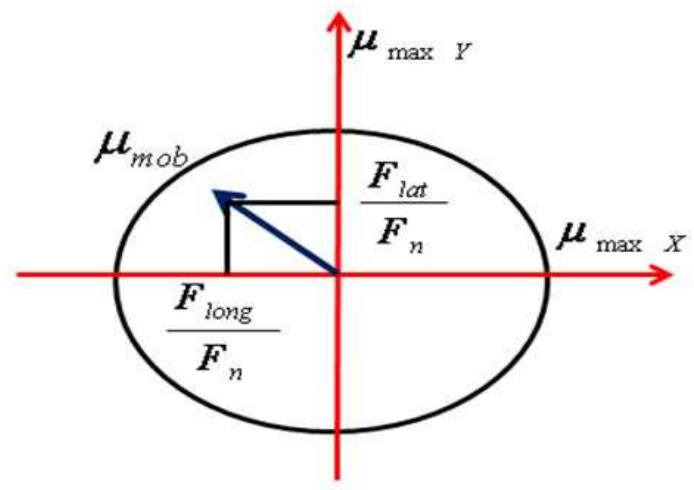

Fig. 3. Friction Ellipse [5]

The Coulomb friction model is used to obtain the mobilized friction $\mu_{m o b}$. Thus the transversal force $F_{t}$ and the normal force $F_{n}=Z_{f}+Z_{r}$ are such that:

$$
\mu_{m o b}=\frac{F_{t}}{F_{n}} \leq \mu_{\text {max }}
$$

Where $\mu_{\max }$ is the maximum available friction. Since: $F_{t}^{2}=F_{\text {lat }}^{2}+F_{\text {long }}^{2}$, we have:

$$
\begin{cases}\mu_{\text {mob }}^{2} & =\frac{F_{\text {lat }}^{2}+F_{\text {long }}^{2}}{F_{n}^{2}}=\mu_{\text {lat }}^{2}+\mu_{\text {long }}^{2} \\ \mu_{\text {lat }} & =\frac{F_{\text {lat }}}{F_{n}} \\ \mu_{\text {long }} & =\frac{F_{\text {long }}}{F_{n}}\end{cases}
$$

Making the following approximations of the yaw rate, lateral and longitudinal accelerations:

$$
\left\{\begin{array}{l}
\gamma_{\text {lat }}=\rho \cdot v_{x}^{2} \\
\gamma_{\text {long }}=\frac{d v_{x}}{d t}=v_{x} \frac{d v_{x}}{d s} \\
\dot{\psi}=\rho \cdot v_{x}
\end{array}\right.
$$

Under the assumption of the low values of the angles (superelevation and slope), and according to (5), (6) and (8) the expressions of the lateral and longitudinal frictions on each wheel are:

$$
\left\{\begin{array}{l}
\mu_{\text {latf }}=\frac{\mu_{\text {lat }}+\left(\frac{I_{z}}{l_{r} M g}\right) \ddot{\psi}}{\cos \phi_{p}-\left(\frac{h F_{\text {long }}}{l_{r} M g}\right)} \\
\mu_{\text {latr }}=\frac{\mu_{\text {lat }}-\left(\frac{I_{z}}{l_{f} M g}\right) \ddot{\psi}}{\cos \phi_{p}+\left(\frac{h F_{\text {long }}}{l_{r} M g}\right)}
\end{array}\right.
$$

with :

$$
\left\{\begin{array}{l}
\mu_{\text {lat }}=\frac{\gamma_{\text {lat }}}{g}+\sin \phi_{r} \\
\mu_{\text {long }}=\frac{\gamma_{\text {long }}}{g}-\sin \phi_{p}
\end{array}\right.
$$

In domain of speed considered, and given sizes of the parameters, we can make the following approximation $\left(\frac{I_{z}}{l_{f} M g}\right)$. $\ddot{\psi}<<\mu_{\text {lat }}$.

Moreover, using approximation of small angles, equation (9) becomes:

$$
\left\{\begin{array}{l}
\mu_{\text {lat }}=\frac{\mu_{\text {lat }}}{1-\left(\frac{h \cdot \gamma_{\text {long }}}{l_{r g}}\right)-\phi_{p}} \\
\mu_{\text {latr }}=\frac{\mu_{\text {lat }}}{1+\left(\frac{h \cdot \gamma_{\text {long }}}{l_{r g}}\right)-\phi_{p}} \\
\mu_{\text {lat }}=\frac{\gamma_{\text {lat }}}{g}+\phi_{r} \\
\mu_{\text {long }}=\frac{\eta_{\text {long }}}{g}-\phi_{p}
\end{array}\right.
$$

The highest safe speed is defined considering the maximum friction. As we consider a two-wheeled vehicle model, we have to find the maximum of the two mobilized frictions, so:

$$
\mu_{\text {max }}=\max \left(\sqrt{\mu_{\text {latf }}^{2}+\mu_{\text {longf }}^{2}}, \sqrt{\mu_{\text {latr }}^{2}+\mu_{\text {longr }}^{2}}\right)
$$

\section{Driver Behaviour}

The driver is unceasingly obliged, according to information which it takes on the environment, to define a speed and a position appropriate to the situation of control [5]. Here the capacities of the driver in acceleration are taking into account. The driver cannot mobilize the same level of acceleration into longitudinal and lateral, and for this reason we have to distinguish between the maximum friction in longitudinal and lateral modes:

$$
\begin{array}{r}
1=\max \left(\sqrt{\left(\frac{\mu_{\text {lat } f}}{\mu_{\text {latmax }}}\right)^{2}+\left(\frac{\mu_{\text {longf }}}{\mu_{\text {longmax }}}\right)^{2}},\right. \\
\left.\sqrt{\left(\frac{\mu_{\text {latr }}}{\mu_{\text {latmax }}}\right)^{2}+\left(\frac{\mu_{\text {longr }}}{\mu_{\text {longmax }}}\right)^{2}}\right)
\end{array}
$$

For comfort and safety reasons, and under good weather conditions, a driver generally does not mobilize all the available lateral and/or longitudinal friction; therefore we represent the driver behaviour with through two adimensional coefficients $\lambda_{\text {lat }}, \lambda_{\text {long }}$ and a maximum friction $\mu_{\max }$ [5].

$$
\mu_{\text {latmax }}=\lambda_{\text {lat }} \cdot \mu_{\max } \text { and } \mu_{\text {longmax }}=\lambda_{\text {long }} \cdot \mu_{\max }
$$

It is well known that driver behaviour adapts according to maximum available friction. We considered that the driver behaviour varies inversely proportional to maximum friction $\mu_{\max }$.

$$
\left\{\begin{array}{l}
\lambda_{\text {long }}=\Delta \lambda_{\text {long }} \cdot \mu_{\text {max }}-\lambda_{\text {slip-long }} \\
\lambda_{\text {lat }}=\Delta \lambda_{\text {lat }} \cdot \mu_{\text {max }}-\lambda_{\text {slip-lat }}
\end{array}\right.
$$

with: 


$$
\left\{\begin{array}{l}
\Delta \lambda_{\text {long }}=\lambda_{\text {dry-long }}-\lambda_{\text {slip-long }} \\
\Delta \lambda_{\text {lat }}=\lambda_{\text {dry-lat }}-\lambda_{\text {slip-lat }}
\end{array}\right.
$$

Where, $\lambda_{d r y}$ (resp. $\lambda_{\text {slip }}$ ) represents the quantity of maximum friction that the driver can mobilize on a dry road (resp. slipping road).

Finally, we get the following expressions for the maximum speed in curve:

In deceleration case,

$$
\begin{aligned}
& 1=\left\{\frac{1}{\lambda_{\text {lat }} \cdot \mu_{\max }}\left(\frac{\frac{\rho v_{x}^{2}}{g}+\phi_{r}}{1+\frac{h}{l_{f}}\left(\frac{v_{x}}{g} \frac{d v_{x}}{d s}-\phi_{p}\right)}\right)\right\}^{2}+ \\
& \left\{\frac{1}{\lambda_{\text {long }} \cdot \mu_{\max }}\left(\frac{v_{x}}{g} \frac{d v_{x}}{d s}-\phi_{p}\right)\right\}^{2}
\end{aligned}
$$

In acceleration case,

$$
\begin{aligned}
& 1=\left\{\frac{1}{\lambda_{\text {lat }} \cdot \mu_{\max }}\left(\frac{\frac{\rho v_{x}^{2}}{g}+\phi_{r}}{1-\frac{h}{l_{r}}\left(\frac{v_{x}}{g} \frac{d v_{x}}{d s}-\phi_{p}\right)}\right)\right\}^{2}+ \\
& \left\{\frac{1}{\lambda_{\text {long }} \cdot \mu_{\max }}\left(\frac{v_{x}}{g} \frac{d v_{x}}{d s}-\phi_{p}\right)\right\}^{2}
\end{aligned}
$$

with following limit conditions:

$$
\left\{\begin{array}{l}
\phi_{p} \geq 0 \\
v_{x}^{2}=\frac{g}{\rho}\left\{\left(1-\frac{h}{l_{f}} \phi_{p}\right) \sqrt{1-\left(\frac{\phi_{p}}{\lambda_{\text {long }} \mu_{\max }}\right)^{2}} \cdot \lambda_{\text {lat }} \cdot \mu_{\text {max }}-\phi_{r}\right\} \\
\phi_{p}<0 \\
v_{x}^{2}=\frac{g}{\rho}\left\{\left(1-\frac{h}{l_{f}} \phi_{p}\right) \sqrt{1+\left(\frac{\phi_{p}}{\lambda_{\text {long }} \mu_{\max }}\right)^{2}} \cdot \lambda_{\text {lat }} \cdot \mu_{\max }-\phi_{r}\right\}
\end{array}\right.
$$

The maximum roll angle authorized, when the motorcycle negotiate a curve, according to the above formulas is:

$$
\phi_{\text {des }}=\arctan \left(\frac{v_{x}^{2} \rho}{g}\right)
$$

where $v_{x}^{2}$ is given by eqaution (18). According (1) and (18) equations, we can express the maximum safe roll angle, needed to negotiate a curve as follow (19):

$$
\left\{\begin{array}{l}
\phi_{p} \geq 0 \\
\phi=\tan ^{-1}\left(\left(1-\frac{h}{l_{f}} \phi_{p}\right) \sqrt{1-\left(\frac{\phi_{p}}{\lambda_{\text {long }} \mu_{\max }}\right)^{2}} \lambda_{\text {lat }} \mu_{\max }-\phi_{r}\right) \\
\phi_{p}<0 \\
\phi=\tan ^{-1}\left(\left(1-\frac{h}{l_{f}} \phi_{p}\right) \sqrt{1+\left(\frac{\phi_{p}}{\lambda_{\text {long }} \mu_{\max }}\right)^{2}} \lambda_{\text {lat }} \mu_{\text {max }}-\phi_{r}\right)
\end{array}\right.
$$

- The term $\left(1-\frac{h}{l_{f}} \phi_{p}\right)$, shows the impact of load transfer and its implication on the limitation of lateral mobilized adhesion.

- The term $\sqrt{1-\left(\frac{\phi_{p}}{\lambda_{\text {long }} \mu_{\max }}\right)^{2}}$ represents the longitudinal mobilized adhesion, in purpose to keeping a constant roll angle. In the next section, we show the effects of different parameters.

\section{PARAMETER EFFECTS ANALYSIS}

This section illustrates the influence of the three parameters of the infrastructure model (the slope, the superelevation and the assumed road friction) and the influence of the load transfer. Results are shown on figure 4.

\section{A. Influence of the Superelevation}

The main purpose of the road superelevation is to counteract the lateral acceleration. In figure 4 , the curve simulated is a left curve $\rho>0$. A left curve normally has a negative superelevation $\phi_{r}<0$. One can see that the roll angle difference at the beginning of the curve can be high: for a superelevation of -10 degrees, the roll angle is -14.2 degrees higher than a superelevation of 0 degrees. At opposite, strongly superelevated curves are known to be very dangerous $\rho>0$ and $\phi_{r}>0$. One can see that the tilt (roll) angle estimate for a superelevation of -10 degrees in a left curve leads to a speed profile -5.488 degrees below the one computed for a 0 degrees.

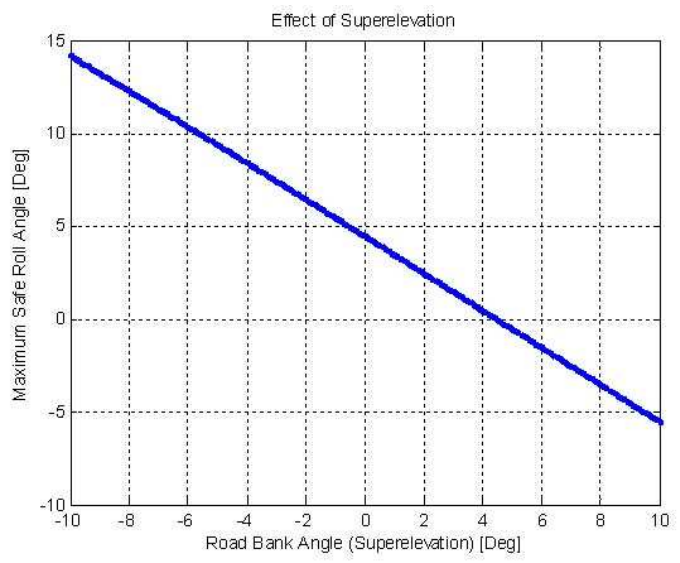

Fig. 4. Effect of the superelevation on the maximum safe roll angle

\section{B. Influence of the Slope}

The second parameter of the infrastructure model is the slope. To keep a constant roll angle (at constant longitudinal speed) in a curve, the driver must accelerate or brake the vehicle according to the sign of the slope, therefore it will mobilize part of maximum available friction. So, maximum safe roll angle (corresponding a maximum speed) in a curve with a slope (positive or negative) is lower than that calculated for a horizontal road. Figure 5 presents the variation of the roll angle profile for different slopes, the safe maximum roll angle decreases as the absolute value of the slope increases.

\section{Influence of the adhesion (friction)}

The third parameter is the road adhesion. In the expression of the boundary conditions equations (18) and (19), the critical roll angle depends on the square root of the maximum adhesion $\mu_{\max }$. Figure 6 represents the variation of the roll angle profile for different adhesion, we can observe that the roll angle is proportional to the maximum road adhesion. 


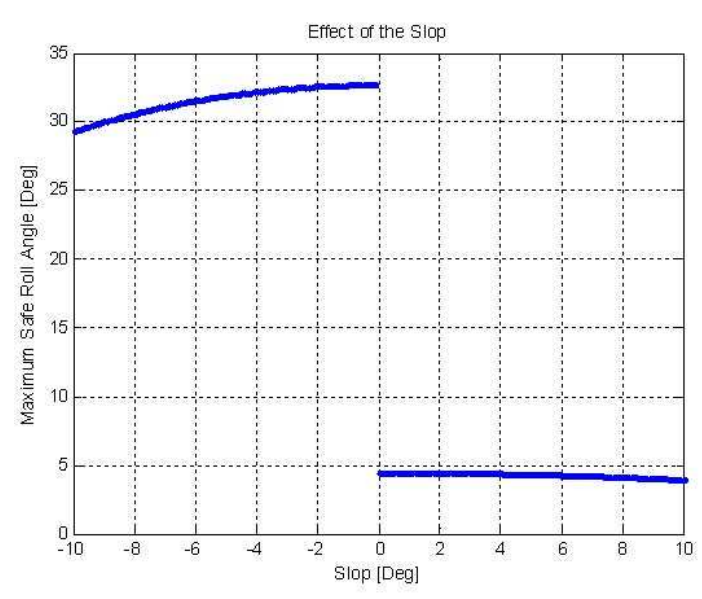

Fig. 5. Effect of the slop on the maximum safe roll angle

In the case of Figure 6, the maximum roll angle obtained by full road adherence using the following simulation parameters

$$
\lambda_{\text {long }}=\lambda_{\text {lat }}=0.4, \phi_{p}=\phi_{r}=0 \Rightarrow \phi=5
$$

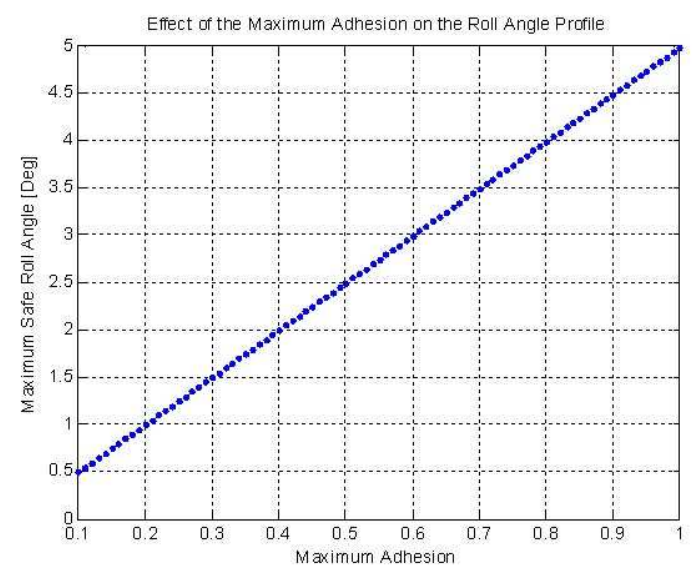

Fig. 6. Effect of the Road Adhesion on the maximum safe roll angle

\section{Influence of the load transfer}

Figure 7 shows the effect of load transfer on the maximum longitudinal acceleration. Using the same conditions as for previous simulations, but with a longitudinal driver capacity, $\lambda_{\text {long }}=1$. The longitudinal deceleration is lower when the load transfer is taken into account. The deceleration decreases the maneuverability of the vehicle. In terms of acceleration, the difference may be close to $50 \%$. This effect has a large influence on the roll angle.

\section{CONCLUSIONS AND FUTURE WORKS}

In this paper, a new method for the calculation of the highest safe roll angle authorized by two-wheelers vehicle (motorcycles) for driving the upcoming curve was proposed. This safe roll angle model takes into account the infrastructure characteristics using an accurate description of the road

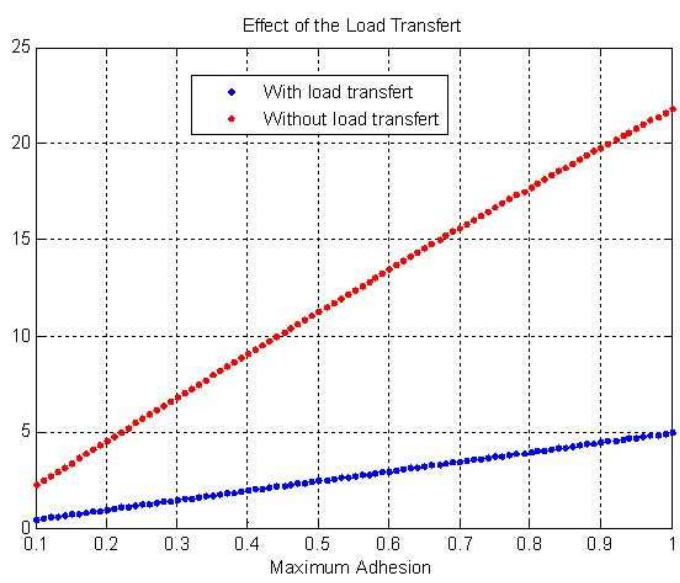

Fig. 7. Effect of the Load Transfer

geometry (radius of curvature, slope and superelevation), and the estimated maximal available friction, the vehicle dynamics and driver behaviour. Parameter effects analysis has been performed. Moreover, the involvement of the vehicle dynamics can be wider, by using observers to estimate the center's gravity of the system, the road adhesion, and the geometry of the road (curvature, road bank and slop angle). These aspects will be incorporated in prototype vehicle and tested.

\section{REFERENCES}

[1] Evangelou, S., "Influence of Road Camber on Motorcycle Stability". Electrical and Electronic and Mechanical Engineering Imperial College London, London SW7 2AZ, U.K.

[2] V. Cossalter and R. Lot, "A motorcycle multi-body model for real time simulations based on the natural coordinates approach,"Vehicle System Dynamics", vol. 37, no. 6, pp. 423-447, 2002. H. Poor, An Introduction to Signal Detection and Estimation. New York: SpringerVerlag, 1985, ch. 4.

[3] D. Karnopp, and R. Hibbard, "Optimum Roll Angle behavior for Tilting Ground Vehicle", DSC, Vol. 44, 1992, pp. 29-37.E. H. Miller, "A note on reflector arrays (Periodical style-Accepted for publication)," IEEE Trans. Antennas Propagat., to be published.

[4] Sang-Gyun So and D. Karnopp, "Methods of Controlling the Lean Angle of Tilting Vehicles", DSC, Vol. 52, 1993, pp. 311-319.C. J. Kaufman, Rocky Mountain Research Lab., Boulder, CO, private communication, May 1995.

[5] Glaser, S., 2004, "Modlisation et analyse d'un vhicule en trajectoire limites Application au dveloppement de systmes d'aide la conduite". $\mathrm{PhD}$ thesis, University of Evry Val d'Essonne (France).M. Young, The Techincal Writers Handbook. Mill Valley, CA: University Science, 1989.

[6] R. S. Sharp and D. J. N. Limebeer, "A motorcycle model for stability and control analysis,"Multibody System Dynamics, vol. 6, no. 2, pp. 123-142, 2001. S. Chen, B. Mulgrew, and P. M. Grant, ”A clustering technique for digital communications channel equalization using radial basis function networks," IEEE Trans. Neural Networks, vol. 4, pp. 570-578, July 1993.

[7] D. J. N. Limebeer and R. S. Sharp, "Bicycles, motorcycles and models," IEEE Control Systems magazine, vol. 26, no. 5, pp. 34-61, 2006.

[8] J. P. Meijaard, J. M. Papadopoulos, A. Ruina, and A. L. Schwab, "Linearized dynamics equations for the balance and steer of a bicycle: a benchmark and review," Proc. R. Soc. A, vol. 463, pp. 19551982, 2007.G. R. Faulhaber, "Design of service systems with priority reservation," in Conf. Rec. 1995 IEEE Int. Conf. Communications, pp. 3-8. 
[9] C. Koenen, "The dynamic behaviour of motorcycles when running straight ahead and when cornering," Ph.D. dissertation, Delft University of Technology, 1983.G. W. Juette and L. E. Zeffanella, "Radio noise currents $\mathrm{n}$ short sections on bundle conductors (Presented Conference Paper style)," presented at the IEEE Summer power Meeting, Dallas, TX, June 22-27, 1990, Paper 90 SM 690-0 PWRS.

[10] S. Kidane, L. Alexander, R. Rajamani, P. Starr and M. Donath, "Road Bank Angle Considerations in Modeling and Tilt Stability Controller Design for Narrow Commuter Vehicles", Department of Mechanical Engineering, University of Minnesota, Minneapolis, USA, June 14-16, 2006.

[11] H. Slimi, H. Arioui, L. Nouveliere and S. Mammar, "Preventive Safety: Warning System for Control Loss of Two-Wheeled Vehicles", Informatics, Integrative Biology and Complex Systems, Zarzis, Tunisia on 23-25 March 2009.

[12] B. Amans, M. Moutreuil, "RIDER : Recherche sur les accidents impliquant un deux roues motoris", Mars 2005.

[13] S Hima, L Nehaoua, N Sguy, H Arioui, "Motorcycle Dynamic Model Synthesis for Two Wheeled Driving Simulator", IEEE Intelligent Transportation Systems Conference, pp. 812-817, 2007. 